ANNALES

POLONICI MATHEMATICI

$92.1(2007)$

\title{
On infinitesimal automorphisms of foliated manifolds
}

\author{
by JAn KureK (Lublin) and WŁodzimierz M. Mikulski (Kraków)
}

\begin{abstract}
Let $F: \mathcal{F}_{O l} \rightarrow \mathcal{F} \mathcal{M}$ be a product preserving bundle functor on the category $\mathcal{F}_{\mathcal{O}}$ of foliated manifolds $(M, \mathcal{F})$ without singularities and leaf respecting maps. We describe all natural operators $C$ transforming infinitesimal automorphisms $X \in \mathcal{X}(M, \mathcal{F})$ of foliated manifolds $(M, \mathcal{F})$ into vector fields $C(X) \in \mathcal{X}(F(M, \mathcal{F}))$ on $F(M, \mathcal{F})$.
\end{abstract}

Introduction. The class of product preserving bundle functors on the category $\mathcal{F}_{o l}$ of foliated manifolds without singularities and their leaf respecting maps is a wide class of bundle functors. For example, the normal bundle functor $N: \mathcal{F}_{O} l \rightarrow \mathcal{F} \mathcal{M}$ sending foliated manifolds $(M, \mathcal{F})$ to their normal bundles $N(M, \mathcal{F})$ and leaf respecting maps $f:\left(M_{1}, \mathcal{F}_{1}\right) \rightarrow\left(M_{2}, \mathcal{F}_{2}\right)$ to the induced maps $N(f): N\left(M_{1}, \mathcal{F}_{1}\right) \rightarrow N\left(M_{2}, \mathcal{F}_{2}\right)$ is product preserving. More generally, for any Weil algebra $A$ the bundle functor $\widetilde{A}: \mathcal{F}$ ol $\rightarrow \mathcal{F M}$ of transverse $A$-points sending foliated manifolds $(M, \mathcal{F})$ to their bundles $\widetilde{A}(M, \mathcal{F})$ of transverse $A$-points in the sense of [5] and leaf respecting maps $f$ : $\left(M_{1}, \mathcal{F}_{1}\right) \rightarrow\left(M_{2}, \mathcal{F}_{2}\right)$ to the induced maps $\widetilde{A}(f): \widetilde{A}\left(M_{1}, \mathcal{F}_{1}\right) \rightarrow \widetilde{A}\left(M_{2}, \mathcal{F}_{2}\right)$ is product preserving. Also, the usual Weil bundle functor $T^{A}: \mathcal{M f} \rightarrow \mathcal{F M}$ on manifolds can be considered as the product preserving bundle functor $T^{A}: \mathcal{F}_{o l} \rightarrow \mathcal{F} \mathcal{M}$ satisfying $T^{A}(M, \mathcal{F})=T^{A} M$ for any foliated manifold $(M, \mathcal{F})$. In particular, the tangent bundle functor on manifolds can be considered as the product preserving bundle functor $T: \mathcal{F} o l \rightarrow \mathcal{F} \mathcal{M}$ satisfying $T(M, \mathcal{F})=T M$ for any foliated manifold $(M, \mathcal{F})$. In [3], the second author described all product preserving bundle functors on the category $\mathcal{F}_{O l}$ in terms of Weil algebra homomorphisms. He deduced

THEOREM A ([3]). There is a bijection between the isomorphism classes of product preserving bundle functors on $\mathcal{F}$ ol and the isomorphism classes of Weil algebra homomorphisms.

2000 Mathematics Subject Classification: 58A05, 58A20.

Key words and phrases: foliated manifolds, (product preserving) bundle functors, natural operators, vector fields, infinitesimal automorphisms of foliated manifolds. 
TheOREM B ([3]). Given two product preserving bundle functors on $\mathcal{F}_{\text {ol }}$ there is a bijection between natural transformations of them and morphisms of the corresponding Weil algebra homomorphisms.

Let $(M, \mathcal{F})$ be a foliated manifold (a $\mathcal{F}$ ol-object). A vector field $X$ on $M$ is called an infinitesimal automorphism of $(M, \mathcal{F})$ if its flow $\operatorname{Exp}(t X)$ is

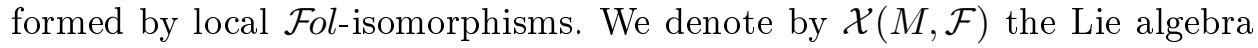
of infinitesimal automorphisms of $(M, \mathcal{F})$.

Let $F: \mathcal{F}_{O} \rightarrow \mathcal{F} \mathcal{M}$ be a product preserving bundle functor. Let $\mu$ : $A \rightarrow B$ be the Weil algebra homomorphism corresponding to $F: \mathcal{F}_{O} l \rightarrow$ $\mathcal{F M}$ (Theorem A). In this paper we study the problem how an infinitesimal automorphism $X \in \mathcal{X}(M, \mathcal{F})$ can induce canonically a vector field $C(X) \in \mathcal{X}(F(M, \mathcal{F}))$ on $F(M, \mathcal{F})$. This problem is reflected in the concept of natural operators $C: \mathcal{X}(M, \mathcal{F}) \rightarrow \mathcal{X}(F(M, \mathcal{F}))$ in the sense of [2]. ( $X$ is an infinitesimal automorphism of $\mathcal{F}$ if its flow preserves $\mathcal{F}$, or equivalently, $[X, Y]$ is tangent to $\mathcal{F}$ for any vector field $Y$ tangent to $\mathcal{F}$.) In the present note we explicitly describe all natural operators $C$ in question. More precisely, we have the flow operator transforming any infinitesimal automorphism $X \in \mathcal{X}(M, \mathcal{F})$ into a vector field $\mathcal{F} X \in \mathcal{X}(F(M, \mathcal{F}))$ with the flow $\operatorname{Exp}(t \mathcal{F} X):=F(\operatorname{Exp}(t X))$. Given $a \in A$ one can define canonically an affinor $a f(a): T F(M, \mathcal{F}) \rightarrow T F(M, \mathcal{F})$ on $F(M, \mathcal{F})$ (see Section 5). Any derivation $D \in \mathcal{D} e r(\mu)=\mathcal{L} i e(\operatorname{Aut}(\mu))$ of $\mu$ yields the corresponding one-parameter group $D_{t}: \mu \rightarrow \mu$ of automorphisms of $\mu$. Then (see Theorem B) we have the corresponding flow $D_{t}: F(M, \mathcal{F}) \rightarrow F(M, \mathcal{F})$ which defines $\operatorname{op}(D) \in \mathcal{X}(F(M, \mathcal{F}))$. Our main result can be stated as follows.

TheOREM C. Let $F: \mathcal{F}_{o l} \rightarrow \mathcal{F} \mathcal{M}$ be a product preserving bundle functor. Let $\mu: A \rightarrow B$ be the Weil algebra homomorphism corresponding to $F$. Let $p \geq 1$ and $q \geq 1$ be integers. Let $\mathcal{F}_{0} l_{p, q}$ be the subcategory of foliated $(p+q)$-dimensional manifolds with $p$-dimensional leaves and their leaf respecting local diffeomorphisms. Any $\mathcal{F}_{\text {ol }}{ }_{p, q}$-natural operator $C$ transforming infinitesimal automorphisms $X \in \mathcal{X}(M, \mathcal{F})$ of $\mathcal{F}_{\text {ol }}, q^{-}$objects into vector fields $C(X) \in \mathcal{X}(F(M, \mathcal{F}))$ on $F(M, \mathcal{F})$ is of the form

$$
C(X)=\operatorname{af}(a) \circ \mathcal{F} X+\mathrm{op}(D)
$$

for some unique $a \in A$ and $D \in \operatorname{Der}(\mu)$.

We remark that Theorem $\mathrm{C}$ is a generalization of the well known result by I. Kolář ([1]) on natural operators lifting vector fields from manifolds to product preserving bundles over manifolds.

All manifolds are assumed to be finite-dimensional. All manifolds and maps are assumed to be smooth, i.e. of class $\mathcal{C}^{\infty}$. All foliations are assumed to be without singularities. 


\section{Product preserving bundle functors on foliated manifolds.} For the reader's convenience we cite (without proofs) some facts from [3]. We start from the following definitions (see e.g. [2]).

Let $F: \mathcal{F}_{o l} \rightarrow \mathcal{F} \mathcal{M}$ be a covariant functor. Let $B_{\mathcal{F M}}: \mathcal{F} \mathcal{M} \rightarrow \mathcal{M f}$ be the base functor and $B_{\mathcal{F}_{o l}}: \mathcal{F}$ ol $\rightarrow \mathcal{M f}$ be the forgetful functor.

A bundle functor on $\mathcal{F}_{O} l$ is a functor $F$ as above satisfying:

(i) (Base preservation) $B_{\mathcal{F} \mathcal{M}} \circ F=B_{\mathcal{F}_{o l}}$. Hence the induced projections form a functor transformation $\pi: F \rightarrow B_{\mathcal{F}_{o l}}$.

(ii) (Localization) For every inclusion $i_{(U, \mathcal{F} \mid U)}:(U, \mathcal{F} \mid U) \rightarrow(M, \mathcal{F})$ of an open subset, $F(U, \mathcal{F} \mid U)$ is the restriction $\pi^{-1}(U)$ of $\pi: F(M, \mathcal{F}) \rightarrow$ $M$ over $U$ and $F i_{(U, \mathcal{F} \mid U)}$ is the inclusion $\pi^{-1}(U) \rightarrow F(M, \mathcal{F})$.

Given two bundle functors $F_{1}, F_{2}$ on $\mathcal{F}_{0}$, by a natural transformation $\nu: F_{1} \rightarrow F_{2}$ we shall mean a system of base preserving fibered maps $\nu$ : $F_{1}(M, \mathcal{F}) \rightarrow F_{2}(M, \mathcal{F})$ for every foliated manifold $(M, \mathcal{F})$ satisfying $F_{2} f \circ \nu=$ $\nu \circ F_{1} f$ for every $\mathcal{F}_{o l}$-morphism $f$.

A bundle functor $F$ on $\mathcal{F}_{0}$ is product preserving if for any product projections $\left(M_{1}, \mathcal{F}_{1}\right) \stackrel{\mathrm{pr}_{1}}{\longleftarrow}\left(M_{1}, \mathcal{F}_{1}\right) \times\left(M_{2}, \mathcal{F}_{2}\right) \stackrel{\mathrm{pr}_{2}}{\longrightarrow}\left(M_{2}, \mathcal{F}_{2}\right)$ (in the category $\left.\mathcal{F}_{\text {ol }}\right), F\left(M_{1}, \mathcal{F}_{1}\right) \stackrel{F p_{1}}{\longleftarrow} F\left(\left(M_{1}, \mathcal{F}_{1}\right) \times\left(M_{2}, \mathcal{F}_{2}\right)\right) \stackrel{F p_{2}}{\longrightarrow} F\left(M_{2}, \mathcal{F}_{2}\right)$ are product projections in the category $\mathcal{F} \mathcal{M}$. In other words, $F\left(\left(M_{1}, \mathcal{F}_{1}\right) \times\left(M_{2}, \mathcal{F}_{2}\right)\right)=$ $F\left(M_{1}, \mathcal{F}_{1}\right) \times F\left(M_{2}, \mathcal{F}_{2}\right)$ modulo $\left(F \mathrm{pr}_{1}, F \mathrm{pr}_{2}\right)$.

Some examples of product preserving bundle functors on $\mathcal{F}_{\text {ol }}$ have been mentioned above. Now, we present the most general example of such a functor.

Let $A$ be an associative algebra over the field $\mathbb{R}$ with unit 1 . The algebra $A$ is called a Weil algebra if it is commutative, of finite dimension over $\mathbb{R}$, and if it admits a unique maximal ideal $\underline{A}$ of codimension 1 such that $\underline{A}^{h+1}=0$ for some non-negative integer $h$.

Let $\mu: A \rightarrow B$ be a homomorphism of Weil algebras. We are going to construct a product preserving bundle functor $T^{\mu}: \mathcal{F}$ ol $\rightarrow \mathcal{F} \mathcal{M}$.

ExAmPlE 1 ([3]). For a foliated manifold $(M, \mathcal{F})$ and $x \in M$ we denote the algebra of germs at $x$ of smooth maps $M \rightarrow \mathbb{R}$ by $C_{x}^{\infty}(M)$, and the algebra of germs at $x$ of smooth maps $M \rightarrow \mathbb{R}$ constant on leaves by $C_{x}^{\infty}(M, \mathcal{F})$. Let $T_{x}^{\mu}(M, \mathcal{F})$ be the set of pairs $(\varphi, \psi)$ of algebra homomorphisms $\varphi: C_{x}^{\infty}(M, \mathcal{F}) \rightarrow A$ and $\psi: C_{x}^{\infty}(M) \rightarrow B$ such that

$$
\psi(u v)=\mu(\varphi(u)) \psi(v)
$$

for any $u \in C_{x}^{\infty}(M, \mathcal{F})$ and any $v \in C_{x}^{\infty}(M)$. Let us define $T^{\mu}(M, \mathcal{F})=$ $\bigcup_{x \in M} T_{x}^{\mu}(M, \mathcal{F})$. Then the obvious projection $\pi: T^{\mu}(M, \mathcal{F}) \rightarrow M$ is a smooth bundle. More precisely, for an adapted chart $\left(x^{1}, \ldots, x^{q}, y^{1}, \ldots, y^{p}\right)$ on $(M, \mathcal{F})$, where $\mathcal{F}$ is $p$-dimensional and $M$ is $p+q$-dimensional, we have a set of induced coordinates $\left(\widetilde{x}^{1}, \ldots, \widetilde{x}^{q}, \widetilde{y}^{1}, \ldots, \widetilde{y}^{p}\right): T^{\mu}(M, \mathcal{F}) \mid U \rightarrow A^{q} \times B^{p}$ 
such that $\widetilde{x}^{i}(\varphi)=\varphi\left(\left[x^{i}\right]_{x}\right) \in A$ and $\widetilde{y}^{j}(\psi)=\psi\left(\left[y^{j}\right]_{x}\right) \in B$ for any $(\varphi, \psi) \in$ $T_{x}^{\mu}(M, \mathcal{F}), x \in M$. (Condition $(*)$ implies that $(\varphi, \psi) \in T_{x}^{\mu}(M, \mathcal{F})$ is uniquely determined by its induced coordinates). Every $\mathcal{F}_{\text {ol-map }} f:\left(M_{1}, \mathcal{F}_{1}\right) \rightarrow$ $\left(M_{2}, \mathcal{F}_{2}\right)$ induces a fibered map $T^{\mu} f: T^{\mu}\left(M_{1}, \mathcal{F}_{1}\right) \rightarrow T^{\mu}\left(M_{2}, \mathcal{F}_{2}\right)$ covering $f$ such that $T^{\mu} f(\varphi, \psi)=(\bar{\varphi}, \bar{\psi})$ for any $(\varphi, \psi) \in T_{x}^{\mu}\left(M_{1}, \mathcal{F}_{1}\right), x \in M_{1}$, where $\bar{\varphi}: C_{f(x)}^{\infty}\left(M_{2}, \mathcal{F}_{2}\right) \rightarrow A$ is defined by $\bar{\varphi}(u)=\varphi(u \circ f)$ and $\bar{\psi}: C_{f(x)}^{\infty}\left(M_{2}\right) \rightarrow B$ is defined by $\bar{\psi}(v)=\psi(v \circ f), u \in C_{f(x)}^{\infty}\left(M_{2}, \mathcal{F}_{2}\right), v \in C_{f(x)}^{\infty}(M)$. If in adapted coordinates a foliated map is of the form $f: \mathbb{R}^{q} \times \mathbb{R}^{p} \rightarrow \mathbb{R}^{\bar{q}} \times \mathbb{R}^{\bar{p}}$, $f\left(x_{i}, y_{j}\right)=\left(f_{1}\left(x_{i}\right), f_{2}\left(x_{i}, y_{j}\right)\right)$ for some $f_{1}: \mathbb{R}^{q} \rightarrow \mathbb{R}^{\bar{q}}$ and $f_{2}: \mathbb{R}^{q} \times \mathbb{R}^{p} \rightarrow \mathbb{R}^{\bar{p}}$, then in the corresponding induced coordinates $T^{\mu} f: A^{q} \times B^{p} \rightarrow A^{\bar{q}} \times B^{\bar{p}}$, $T^{\mu}\left(a_{i}, b_{j}\right)=\left(T_{A} f_{1}\left(a_{i}\right), T_{B} f_{2}\left(\mu\left(a_{i}\right), b_{j}\right)\right)$, where $T_{A}: \mathcal{M} f \rightarrow \mathcal{F} \mathcal{M}$ is the Weil functor [2]. The correspondence $T^{\mu}: \mathcal{F}_{O} l \rightarrow \mathcal{F} \mathcal{M}$ is a product preserving bundle functor. It is called the product preserving bundle functor on $\mathcal{F}_{o l}$ corresponding to the Weil algebra homomorphism $\mu$.

REMARK 1. Let us observe that given a $p$-dimensional foliation $\mathcal{F}$ on a $p+q$-dimensional manifold $M$ the bundle $T^{\mu}(M, \mathcal{F})$ admits a canonical $p \operatorname{dim}_{\mathbb{R}} B$-dimensional foliation $\mathcal{F}^{\mu} \pi$-related to $\mathcal{F}$. In the induced coordinates the leaves of $\mathcal{F}^{\mu}$ are of the form $\{a\} \times B^{p} \subset A^{q} \times B^{p}$.

Let $F: \mathcal{F}_{o l} \rightarrow \mathcal{F M}$ be a product preserving bundle functor. We are going to construct a Weil algebra homomorphism $\mu^{F}: A^{F} \rightarrow B^{F}$.

EXAMPLE $2([3])$. We put $A^{F}=F\left(\mathbb{R}, \mathcal{F}^{\prime}\right)$ and $B^{F}=F\left(\mathbb{R}, \mathcal{F}^{\prime \prime}\right)$, where $\mathcal{F}^{\prime}$ is the 0 -dimensional foliation on $\mathbb{R}$ and $F^{\prime \prime}$ is the foliation on $\mathbb{R}$ with one leaf $\mathbb{R}$. The sum mappings are given by $+_{A^{F}}=F(+): A^{F} \times A^{F} \rightarrow A^{F}$ and $+_{B^{F}}=F(+): B^{F} \times B^{F} \rightarrow B^{F}$, where $+: \mathbb{R} \times \mathbb{R} \rightarrow \mathbb{R}$ is the sum map treated as the respective $\mathcal{F}_{\text {ol-morphisms. Similarly, the multiplications }}$ of $A^{F}$ and $B^{F}$ are obtained by applying $F$ to the multiplication of $\mathbb{R}$ being

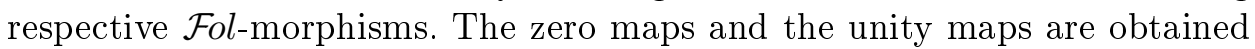
by applying $F$ to the zero map and the unity map of $\mathbb{R}$. The homomorphism $\mu^{F}: A^{F} \rightarrow B^{F}$ is $F\left(\operatorname{id}_{\mathbb{R}}\right): F\left(\mathbb{R}, \mathcal{F}^{\prime}\right) \rightarrow F\left(\mathbb{R}, \mathcal{F}^{\prime \prime}\right)$, where $i_{\mathbb{R}}$ is the identity map of $\mathbb{R}$ treated as the appropriate $\mathcal{F}_{\text {ol-morphism. }}{ }^{*}$

For example, the Weil algebra homomorphism corresponding to the normal bundle functor $N: \mathcal{F}$ ol $\rightarrow \mathcal{F} \mathcal{M}$ (see Introduction) is the unique algebra homomorphism $\kappa_{\mathbb{D}}: \mathbb{D} \rightarrow \mathbb{R}$, where $\mathbb{D}=\mathbb{R} \times \mathbb{R}$ is the Weil algebra of dual numbers $((a, b)+(c, d)=(a+c, b+d),(a, b)(c, d)=(a c, a d+b c)$ for $(a, b),(c, d) \in \mathbb{D})$. The Weil algebra homomorphism corresponding to the bundle functor $\widetilde{A}: \mathcal{F}$ ol $\rightarrow \mathcal{F} \mathcal{M}$ of transverse $A$-points (mentioned in Introduction) is the unique algebra homomorphism $\kappa_{A}: A \rightarrow \mathbb{R}$. The Weil algebra homomorphism corresponding to the product preserving bundle functor $T^{A}: \mathcal{F}_{O l} \rightarrow \mathcal{F} \mathcal{M}$ of $A$-near points (see Introduction) is the identity map $\operatorname{id}_{A}: A \rightarrow A$. 
The following classification proposition shows that any product preserving bundle functor on $\mathcal{F}_{o l}$ is equivalent to some product preserving bundle functor as in Example 1.

Proposition 1 ([3]). Let $F: \mathcal{F}_{\text {Ol }} \rightarrow \mathcal{F} \mathcal{M}$ be a product preserving bundle functor. Let $\mu^{F}: A^{F} \rightarrow B^{F}$ be the corresponding Weil algebra homomorphism. Then we have an explicitly constructed natural equivalence $\Theta^{F}: F \cong T^{\mu^{F}}$.

More precisely, the equivalence $\Theta^{F}: F(M, \mathcal{F}) \rightarrow T^{\mu^{F}}(M, \mathcal{F})$ is the following. Given a point $y \in F_{x}(M, \mathcal{F}), x \in M$, we define $\Theta^{F}(y)=\left(\varphi_{y}, \psi_{y}\right)$, where $\varphi_{y}: C_{x}^{\infty}(M, \mathcal{F}) \rightarrow A^{F}, \psi_{y}: C_{x}^{\infty}(M) \rightarrow B^{F}, \varphi_{y}\left([f]_{x}\right)=F f(y)$, $\psi_{y}\left([g]_{x}\right)=F g(y)$ for $[f]_{x} \in C_{x}^{\infty}(M, \mathcal{F})$ and $[g]_{x} \in C_{x}^{\infty}(M)$, where $f$ and $g$ are treated as the corresponding $\mathcal{F}_{\text {ol }}$-morphisms. Using the respective definitions, $\Theta^{F}(y) \in T_{x}^{\mu^{F}}(M, \mathcal{F})$. In [3], it is proved that $\Theta^{F}: F(M, \mathcal{F}) \rightarrow$ $T^{\mu^{F}}(M, \mathcal{F})$ is a diffeomorphism.

REMARK 2. Let $F: \mathcal{F}_{O} l \rightarrow \mathcal{F} \mathcal{M}$ be a product preserving bundle functor. By Proposition 1 we have the canonical diffeomorphism $\Theta^{F}: F(M, \mathcal{F}) \rightarrow$ $T^{\mu^{F}}(M, \mathcal{F})$ for any foliated manifold $(M, \mathcal{F})$. It is known that $T^{\mu^{F}}(M, \mathcal{F})$ admits the foliation $\mathcal{F}^{\mu^{F}}$ (see Remark 1). Then $F(M, \mathcal{F})$ admits the foliation $\mathcal{F}^{F}=\left(\Theta^{F}\right)^{-1} \mathcal{F}^{\mu^{F}}$. Hence $F$ has values in the category $\mathcal{F}$ ol. Therefore we can compose two product preserving bundle functors on $\mathcal{F}_{o l}$. This composition is again a product preserving bundle functor on $\mathcal{F}_{o l}$.

The following proposition shows that any Weil algebra homomorphism is isomorphic to the Weil algebra homomorphism corresponding to some product preserving bundle functor on $\mathcal{F}_{O}$.

Proposition 2 ([3]). Let $\mu: A \rightarrow B$ be a Weil algebra homomorphism. Let $F=T^{\mu}$. Then we have an explicitly constructed isomorphism $\mathcal{O}^{\mu}: \mu \cong \mu^{F}$ of Weil algebra homomorphisms.

We recall that a morphism $\mu_{1} \rightarrow \mu_{2}$ of Weil algebra homomorphisms $\mu_{1}: A_{1} \rightarrow B_{1}$ and $\mu_{2}: A_{2} \rightarrow B_{2}$ is a pair $\eta$ of Weil algebra homomorphisms $\eta_{1}: A_{1} \rightarrow A_{2}$ and $\eta_{2}: B_{1} \rightarrow B_{2}$ such that $\eta_{2} \circ \mu_{1}=\mu_{2} \circ \eta_{1}$.

More precisely, $\mathcal{O}^{\mu}: \mu \rightarrow \mu^{F}$ is the pair of Weil algebra isomorphisms $\mathcal{O}_{1}^{\mu}: A \cong T^{\mu}\left(\mathbb{R}, \mathcal{F}^{\prime}\right) \rightarrow F\left(M, \mathcal{F}^{\prime}\right)=A^{F}$ and $\mathcal{O}_{2}^{\mu}: B \cong T^{\mu}\left(\mathbb{R}, \mathcal{F}^{\prime \prime}\right) \rightarrow$ $F\left(\mathbb{R}, \mathcal{F}^{\prime \prime}\right)=B^{F}$, where $\cong$ is the induced coordinates $\widetilde{x}$ and $\widetilde{y}$ (see Example 1 ) respectively.

Let $F_{1}, F_{2}: \mathcal{F}_{0 l} \rightarrow \mathcal{F} \mathcal{M}$ be product preserving bundle functors. Let $\mu^{F_{1}}: A^{F_{1}} \rightarrow B^{F_{1}}$ and $\mu^{F_{2}}: A^{F_{2}} \rightarrow B^{F_{2}}$ be the corresponding Weil algebra homomorphisms. Let $\nu: F_{1} \rightarrow F_{2}$ be a natural transformation.

ExAmple $3([3])$. Define a morphism $\eta^{\nu}=\left(\eta_{1}^{\nu}, \eta_{2}^{\nu}\right): \mu^{F_{1}} \rightarrow \mu^{F_{2}}$ of Weil algebra homomorphisms by $\eta_{1}^{\nu}=\nu_{\left(\mathbb{R}, \mathcal{F}^{\prime}\right)}: A^{F_{1}} \rightarrow A^{F_{2}}$ and $\eta_{2}^{\nu}=\nu_{\left(\mathbb{R}, \mathcal{F}^{\prime \prime}\right)}$ : 
$B^{F_{1}} \rightarrow B^{F_{2}}$. If $\nu$ is an isomorphism, then so is $\eta^{\nu}$. We call $\eta^{\nu}$ the morphism of Weil algebra homomorphisms corresponding to $\nu$.

Let $\mu_{1}: A_{1} \rightarrow B_{1}$ and $\mu_{2}: A_{2} \rightarrow B_{2}$ be Weil algebra homomorphisms. Let $\eta=\left(\eta_{1}, \eta_{2}\right): \mu_{1} \rightarrow \mu_{2}$ be a morphism of Weil algebra homomorphisms.

ExAmple $4([3])$. Given a $\mathcal{F}$ ol-object $(M, \mathcal{F})$ define a base preserving fibered map $\nu^{\eta}: T^{\mu_{1}}(M, \mathcal{F}) \rightarrow T^{\mu_{2}}(M, \mathcal{F})$ by $\nu^{\eta}(\varphi, \psi)=\left(\eta_{1} \circ \varphi, \eta_{2} \circ \psi\right)$, $(\varphi, \psi) \in T_{x}^{\mu_{1}}(M, \mathcal{F}), x \in M$. The family $\nu^{\eta}: T^{\mu_{1}} \rightarrow T^{\mu_{2}}$ is a natural transformation. If $\eta$ is an isomorphism, then so is $\nu^{\eta}$. We call $\nu^{\eta}$ the natural transformation corresponding to $\nu$.

Summing up we have the following object classification theorem corresponding to Theorem A.

THEOREM $1([3])$. The correspondence $F \mapsto \mu^{F}$ induces a bijective correspondence between the equivalence classes of product preserving bundle functors on $\mathcal{F}_{\text {ol }}$ and the equivalence classes of Weil algebra homomorphisms. The inverse correspondence is induced by the correspondence $\mu \mapsto T^{\mu}$.

Let $F_{1}$ and $F_{2}$ be two product preserving bundle functors on $\mathcal{F}_{o l}$. Let $\mu^{F_{1}}: A^{F_{1}} \rightarrow B^{F_{1}}$ and $\mu^{F_{2}}: A^{F_{2}} \rightarrow B^{F_{2}}$ be the corresponding Weil algebra homomorphisms.

LEMMA $1([3])$. Let $\eta=\left(\eta_{1}, \eta_{2}\right): \mu^{F_{1}} \rightarrow \mu^{F_{2}}$ be a morphism of Weil algebra homomorphisms. Let $\nu^{[\eta]}: F_{1} \rightarrow F_{2}$ be a natural transformation given by the composition $F_{1} \stackrel{\Theta^{F_{1}}}{\longrightarrow} T^{\mu^{F_{1}}} \stackrel{\nu^{\eta}}{\longrightarrow} T^{\mu^{F_{2}}} \stackrel{\left(\Theta^{F_{2}}\right)^{-1}}{\longrightarrow} F_{2}$, where $\Theta^{F}$ is as in Proposition 1 and $\nu^{\eta}$ is described in Example 4. Then $\nu=\nu^{[\eta]}$ is the unique natural transformation $F_{1} \rightarrow F_{2}$ such that $\eta^{\nu}=\eta$, where $\eta^{\nu}$ is as in Example 3.

Summing up we have the following morphism classification theorem corresponding to Theorem $\mathrm{B}$.

THEOREM $2([3])$. Let $F_{1}$ and $F_{2}$ be two product preserving bundle functors on $\mathcal{F}_{\text {ol }}$. The correspondence $\nu \mapsto \eta^{\nu}$ is a bijection between natural transformations $F_{1} \rightarrow F_{2}$ and morphisms $\mu^{F_{1}} \rightarrow \mu^{F_{2}}$ of the corresponding Weil algebra homomorphisms. The inverse correspondence is $\eta \mapsto \nu^{[\eta]}$ (where $\nu^{[\eta]}$ is defined in Lemma 1).

Let $F_{1}$ and $F_{2}$ be product preserving bundle functors on $\mathcal{F}_{o}$. According to Remark 2, the composition $F_{1} \circ F_{2}$ is again a product preserving bundle functor on $\mathcal{F}_{o l}$. Let $\mu^{F_{1}}, \mu^{F_{2}}$ and $\mu^{F_{1} \circ F_{2}}$ be the corresponding Weil algebra homomorphisms. Using the tensor product we get the Weil algebra homomorphism $\mu^{F_{1}} \otimes \mu^{F_{2}}$.

Proposition $3([3]) . \mu^{F_{1} \circ F_{2}}=\mu^{F_{1}} \otimes \mu^{F_{2}}$. 
Corollary 1 ([3]). We have the isomorphism $F_{1} \circ F_{2} \cong F_{2} \circ F_{1}$ corresponding to the exchange isomorphism of tensor products.

Corollary 2. Let $F: \mathcal{F}$ ol $\rightarrow \mathcal{F M}$ be a product preserving bundle functor. Let $T: \mathcal{F}$ ol $\rightarrow \mathcal{F} \mathcal{M}$ be the tangent bundle functor. For any $\mathcal{F}_{\text {ol }}$ object $(M, \mathcal{F})$ we have the $\mathcal{F}$ ol-natural isomorphism $\nu_{(M, \mathcal{F})}: F(T(M, \mathcal{F})) \rightarrow$ $T(F(M, \mathcal{F})$ ) (see Corollary 1$)$ satisfying the flow property

$$
\pi^{T(F(M, \mathcal{F}))} \circ \nu_{(M, \mathcal{F})}=F \pi^{T(M, \mathcal{F})}
$$

where $\pi^{T(F(M, \mathcal{F}))}: T(F(M, \mathcal{F})) \rightarrow F(M, \mathcal{F})$ and $\pi^{T(M, \mathcal{F})}: T(M, \mathcal{F}) \rightarrow$ $(M, \mathcal{F})$ are the projections of the tangent bundles (with the respective foliations as in Remark 2).

2. The Lie algebra of $\operatorname{Aut}(\mu)$. Consider a Weil algebra homomorphism $\mu: A \rightarrow B$. We note that the group $\operatorname{Aut}(\mu)$ of all automorphisms of $\mu$ is a closed (and hence Lie) subgroup in $\mathrm{GL}(A) \times \mathrm{GL}(B)$.

We have the Lie algebra

$$
\operatorname{Der}(\mu)=\left\{D=\left(D_{1}, D_{2}\right) \in \operatorname{Der}(A) \times \operatorname{Der}(B) \mid D_{2} \circ \mu=\mu \circ D_{1}\right\}
$$

of derivations of $\mu$. It is a subalgebra in the product $\operatorname{Der}(A) \times \operatorname{Der}(B)$ of the Lie algebras $\operatorname{Der}(A)$ and $\operatorname{Der}(B)$ of derivations of $A$ and $B$. A derivation of $A$ is a linear map $D_{1}: A \rightarrow A$ such that $D_{1}(a b)=D_{1}(a) b+a D_{1}(b)$ for all $a, b \in A$. The bracket of the Lie algebra $\operatorname{Der}(A)$ is given standardly by $\left[D_{1}, D_{1}^{\prime}\right]=D_{1} \circ D_{1}^{\prime}-D_{1}^{\prime} \circ D_{1}$.

Proposition 4 ([4]). $\mathcal{L} i e(\operatorname{Aut}(\mu))=\operatorname{Der}(\mu)$.

Proof. By [2], Lie $(\operatorname{Aut}(A))=\operatorname{Der}(A)$. Clearly, $\left(D_{1}, D_{2}\right) \in \operatorname{Aut}(\mu)$ iff $D_{1} \in \operatorname{Aut}(A), D_{2} \in \operatorname{Aut}(B)$ and $\mu \circ D_{1}=D_{2} \circ \mu$.

3. Natural automorphisms of $F_{\mid \mathcal{F}_{o l_{p, q}}} \rightarrow \mathcal{F} \mathcal{M}$ into itself. In this section we prove the following result.

Proposition 5. Let $F: \mathcal{F}_{\text {ol }} \rightarrow \mathcal{F} \mathcal{M}$ be a product preserving bundle functor and $\mu: A \rightarrow B$ be its algebra homomorphism. Let $p \geq 1$ and $q \geq 1$ be integers. Every natural automorphism $\nu$ of $F_{\mid \mathcal{F}_{o l}, q}$ can be extended uniquely to a natural automorphism of $F$. In particular, $\operatorname{Aut}\left(F_{\mid \mathcal{F}_{o l}, q}\right)=\operatorname{Aut}(\mu)$.

Proof. Let $x^{1}, \ldots, x^{q}, y^{1}, \ldots, y^{p}$ be the adapted coordinates on the stan$\operatorname{dard} \mathcal{F}_{o l} l_{p}$ object $\left(M_{0}, \mathcal{F}_{0}\right)=\left(\mathbb{R}^{q} \times \mathbb{R}^{p},\left\{\{a\} \times \mathbb{R}^{p}\right\}_{a \in \mathbb{R}^{q}}\right)$.

Consider a natural automorphism $\nu$ of $F_{\mid \mathcal{F}_{o l} l_{p, q}}$ into itself. Since $F$ is product preserving, $F\left(M_{0}, \mathcal{F}_{0}\right)=A^{q} \times B^{p}$. By the $\mathcal{F}_{o} l_{p, q}$-naturality, $\nu$ is uniquely determined by $\nu=\nu_{\left(M_{0}, \mathcal{F}_{0}\right)}: A^{q} \times B^{p} \rightarrow A^{q} \times B^{p}$. Write

$$
\nu\left(a_{1}, \ldots, a_{q}, a_{q+1}, \ldots, a_{q+p}\right)=\left(\nu^{1}\left(a_{1}, \ldots, a_{q+p}\right), \ldots, \nu^{q+p}\left(a_{1}, \ldots, a_{q+p}\right)\right)
$$

for $a_{1}, \ldots, a_{q} \in A$ and $a_{q+1}, \ldots, a_{q+p} \in B$. 
By the invariance of $\nu$ with respect to the $\mathcal{F}_{o l}, q^{- \text {morphisms }}\left(\tau_{1} x^{1}, \ldots\right.$, $\left.\tau_{q} x^{q}, \tau_{q+1} y^{1}, \ldots, \tau_{q+p} y^{p}\right)$ for $\tau_{1}, \ldots, \tau_{q+p} \in \mathbb{R}_{+}$we get the homogeneity conditions $\tau_{j} \nu^{j}\left(a_{1}, \ldots, a_{q+p}\right)=\nu^{j}\left(\tau_{1} a_{1}, \ldots, \tau_{q+p} a_{q+p}\right)$ for $j=1, \ldots, q+p$ and any $a_{1}, \ldots, a_{q} \in A, a_{q+1}, \ldots, a_{q+p} \in B$ and any $\tau_{1}, \ldots, \tau_{q+p} \in \mathbb{R}_{+}$. This type of homogeneity implies that $\nu^{j}$ depends linearly only on $a_{j}$ because of the homogeneous function theorem ([2]).

Using permutations of adapted coordinates we deduce that $\nu=\sigma \times \cdots \times$ $\sigma \times \varrho \times \cdots \times \varrho: A^{q} \times B^{p} \rightarrow A^{q} \times B^{p}$ for $\sigma=\nu^{1}: A \rightarrow A$ and $\varrho=\nu^{q+1}: B \rightarrow B$. We prove that $(\sigma, \varrho) \in \operatorname{Aut}(\mu)$.

STEP 1: $\varrho$ is an algebra isomorphism. We know that $\varrho$ is $\mathbb{R}$-linear. Using the invariance of $\nu$ with respect to the local $\mathcal{F}_{o} l_{p, q}$-morphism $\left(x^{1}, \ldots, x^{q}\right.$, $\left.y^{1}+\left(y^{1}\right)^{2}, y^{2}, \ldots, y^{p}\right)$ we derive that $\varrho\left(b+b^{2}\right)=\varrho(b)+(\varrho(b))^{2}$, i.e. $\varrho\left(b^{2}\right)=$ $(\varrho(b))^{2}$ for any $b \in B$. Then $\varrho\left(\left(b_{1}+b_{2}\right)^{2}\right)=\left(\varrho\left(b_{1}+b_{2}\right)\right)^{2}$, i.e. $\varrho\left(b_{1} b_{2}\right)=$ $\varrho\left(b_{1}\right) \varrho\left(b_{2}\right)$ for any $b_{1}, b_{2} \in B$. So, $\varrho$ is multiplicative. Using the invariance of $\nu$ with respect to the $\mathcal{F}_{o} l_{p, q^{-}}$map $\left(x^{1}, \ldots, x^{q}, y^{1}+1, y^{2}, \ldots, y^{p}\right)$ we derive that $\varrho(b+1)=\varrho(b)+1$, i.e. $\varrho(1)=1$. These facts show that $\varrho$ is an algebra homomorphism. Since $\eta$ is an isomorphism, so is $\varrho$.

STEP 2: $\sigma$ is an algebra homomorphism. The proof is quite similar to Step 1. (Now, we use the $\mathcal{F}_{o} l_{p, q^{-m a p}}\left(x^{1}+\left(x^{1}\right)^{2}, x^{1}, \ldots, x^{q}, y^{1}, \ldots, y^{p}\right)$.)

STEP 3: $\varrho \circ \mu=\mu \circ \sigma$. We use the invariance of $\nu$ with respect to the $\mathcal{F}_{o} l_{p, q^{-m a p}} \varphi=\left(x^{1}, \ldots, x^{q}, y^{1}+x^{1}, y^{2}, \ldots, y^{p}\right)$. Then

$$
\begin{aligned}
(\sigma(a), 0, \ldots, 0, \mu(\sigma(a)), 0, \ldots, 0) & =F \varphi \circ \nu(a, 0, \ldots, 0,0, \ldots, 0) \\
& =\nu \circ F \varphi(a, 0, \ldots, 0,0, \ldots, 0) \\
& =(\sigma(a), 0, \ldots, 0, \varrho(\mu(a)), 0, \ldots, 0) .
\end{aligned}
$$

Hence $\varrho(\mu(a))=\mu(\sigma(a))$ for $a \in A$.

We have proved that $\eta=(\sigma, \varrho) \in \operatorname{Aut}(\mu)$. By Theorem 2 we have the natural transformation $\nu^{[\eta]}: F \rightarrow F$ corresponding to $\eta$. Clearly, $\nu$ is the restriction of $\nu^{[\eta]}$. If $\widetilde{\nu}: F \rightarrow F$ is another such transformation, then $\widetilde{\nu}=\nu^{[\eta]}$ because $\widetilde{\nu}$ coincides with $\nu$ on $A^{p} \times B^{q}$.

\section{Canonical vector fields on product preserving bundles over} foliated manifolds. Let $F: \mathcal{F}_{O} l \rightarrow \mathcal{F} \mathcal{M}$ be a product preserving bundle functor and let $\mu: A \rightarrow B$ be its corresponding Weil algebra homomorphism. Let $p \geq 1$ and $q \geq 1$ be integers.

A $\mathcal{F}_{o l} l_{p, q^{-}}$canonical vector field on $F_{\mid \mathcal{F}_{o l_{p, q}}}$ is a family of vector fields $V=$ $V_{F(M, \mathcal{F})}$ on $F(M, \mathcal{F})$ for any $\mathcal{F}_{o l} l_{p, q^{-o b j e c t}}(M, \mathcal{F})$ such that for any $\mathcal{F}_{o l} l_{p, q^{-}}$ $\operatorname{objects}\left(M_{1}, \mathcal{F}_{1}\right)$ and $\left(M_{2}, \mathcal{F}_{2}\right)$ and any $\mathcal{F}_{o l}, q^{-m a p} \varphi:\left(M_{1}, \mathcal{F}_{1}\right) \rightarrow\left(M_{2}, \mathcal{F}_{2}\right)$ the vector fields $V$ on $F\left(M_{1}, \mathcal{F}_{1}\right)$ and $V$ on $F\left(M_{2}, \mathcal{F}_{2}\right)$ are $F \varphi$-related.

We have the following example of canonical vector fields on $F_{\mid \mathcal{F}_{o l_{p, q}}}$. 
Example 5 (The operators op $(D))$. Let $D \in \operatorname{Der}(\mu)=\mathcal{L} i e(\operatorname{Aut}(\mu))$ be an element from the Lie algebra of the Lie group of all automorphisms of $\mu$ (see Proposition 2). Let $D_{t}$ be the one-parameter subgroup in $\operatorname{Aut}(\mu)$ corresponding to $D$. By Theorem 2 we have the corresponding one-parameter subgroup $D_{t}$ of natural equivalences of $F$. So, for every $\mathcal{F}_{\text {ol }}$, $q^{\text {-object }}(M, \mathcal{F})$ we have the flow $D_{t}$ on $F(M, \mathcal{F})$. This flow defines a vector field $\operatorname{op}(D)$ on $F(M, \mathcal{F})$. Clearly, op $(D)$ is canonical.

For example, let $F=N: \mathcal{F}_{o l} l_{p, q} \rightarrow \mathcal{F} \mathcal{M}$ be the normal bundle functor. As we mentioned, the Weil algebra homomorphism $\mu$ corresponding to $N$ is the unique algebra homomorphism $\kappa_{\mathbb{D}}: \mathbb{D} \rightarrow \mathbb{R}$, where $\mathbb{D}=\mathbb{R} \times \mathbb{R}$ is the algebra of dual numbers, $(a, b)+(c, d)=(a+c, b+d),(a, b)(c, d)=(a c, a d+b c)$. Then (it is easy to see that) $\operatorname{Aut}\left(\kappa_{\mathbb{D}}\right)=\left\{\left(\operatorname{id}_{\mathbb{R}} \times \tau \operatorname{id}_{\mathbb{R}}, \operatorname{id}_{\mathbb{R}}\right) \mid \tau \in \mathbb{R} \backslash\{0\}\right\} \cong \mathbb{R} \backslash\{0\}$. Then $\operatorname{Der}\left(\kappa_{D}\right) \cong \mathbb{R}$. For $D=1 \in \mathbb{R}=\operatorname{Der}\left(\kappa_{\mathbb{D}}\right)$, the one-parameter group corresponding to $D=1$ is $D_{t}=\left(\operatorname{id}_{\mathbb{R}} \times \exp (t) \operatorname{id}_{\mathbb{R}}, \operatorname{id}_{\mathbb{R}}\right) \in \operatorname{Aut}\left(\kappa_{\mathbb{D}}\right)$. Then $\operatorname{op}(D)=L$ is the Liouville vector field on the vector bundle $N(M, \mathcal{F})$ for any foliated manifold $(M, \mathcal{F})$ (here $L(v)=[v+t v] \in T_{v} N_{x}(M, \mathcal{F}) \subset T_{v} N(M, \mathcal{F})$, $\left.v \in N_{x}(M, \mathcal{F}), x \in M\right)$.

Proposition 6. Let $F: \mathcal{F}_{\text {ol }} \rightarrow \mathcal{F} \mathcal{M}$ be a product preserving bundle functor and $\mu: A \rightarrow B$ be its Weil algebra homomorphism. Let $p \geq 1$ and $q \geq 1$ be integers. Every $\mathcal{F}_{\text {ol }}, q$-canonical vector field $V$ on $F_{\mid \mathcal{F}_{o l}, q}$ is of the form $V=\mathrm{op}(D)$ for some $D \in \operatorname{Der}(\mu)$.

Proof. For every $\mathcal{F}_{\text {ol }}, q^{\text {-object }}(M, \mathcal{F})$ we have some vector field $V$ on $F(M, \mathcal{F})$ invariant with respect to $\mathcal{F}_{o} l_{p, q}$-maps. The flow $\operatorname{Exp}(t V)$ of $V$ is $\mathcal{F}_{0} l_{p, q}$-invariant. Using Theorem 1 we can easily show that there exists $v \in$ $F(M, \mathcal{F})$ such that $F(M, \mathcal{F})$ is the orbit of $U$ with respect to $\mathcal{F}_{o l} l_{p, q}$-maps for any open neighborhood $U \subset F(M, \mathcal{F})$ of $v$. This implies that $V$ is complete. Thus $\operatorname{Exp}(t V)$ corresponds to some one-parameter subgroup in $\operatorname{Aut}\left(F_{\mathcal{F}_{o l}, q}\right)$. By Proposition 4 and Theorem 2 it corresponds to some $D \in \mathcal{D} e r(\mu)$. Then $V=\mathrm{op}(D)$.

\section{Some canonical affinors on product preserving bundle func-} tors. Let $F: \mathcal{F}_{O l} \rightarrow \mathcal{F} \mathcal{M}$ be a product preserving bundle functor and $\mu: A \rightarrow B$ be its Weil algebra homomorphism.

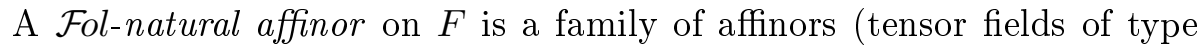
$(1,1)) t=t_{F(M, \mathcal{F})}$ on $F(M, \mathcal{F})$ for any $\mathcal{F}$ ol-object $(M, \mathcal{F})$ such that for any $\mathcal{F}$ ol-map $\varphi:\left(M_{1}, \mathcal{F}_{1}\right) \rightarrow\left(M_{2}, \mathcal{F}_{2}\right)$ the tensor fields $t$ on $F\left(M_{1}, \mathcal{F}_{1}\right)$ and $t$ on $F\left(M_{2}, \mathcal{F}_{2}\right)$ are $F \varphi$-related.

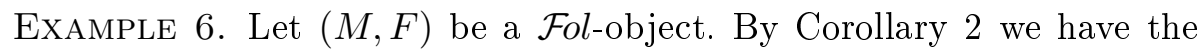
natural isomorphism $\nu_{(M, \mathcal{F})}: F(T(M, \mathcal{F})) \rightarrow T(F(M, \mathcal{F}))$ satisfying the flow property. The fiber multiplication $m: \mathbb{R} \times T(M, \mathcal{F}) \rightarrow T(M, \mathcal{F})$ can be treated as a $\mathcal{F}$ ol-map, where $\mathbb{R}$ is foliated by points and $T(M, \mathcal{F})$ is a 
foliated manifold (see Remark 2). Applying the functor $F$ we obtain $F m$ : $F \mathbb{R} \times F(T(M, \mathcal{F})) \rightarrow F(T(M, \mathcal{F}))$. Using the above flow isomorphism and observing that $F \mathbb{R}=A$ (see Example 2) from $F m$ we obtain $\widetilde{F m}: A \times$ $T(F(M, \mathcal{F})) \rightarrow T(F(M, \mathcal{F}))$. Thus given $a \in A$ we have

$$
\operatorname{af}(a):=\widetilde{F m}(a, \cdot): T(F(M, \mathcal{F})) \rightarrow T(F(M, \mathcal{F})) .
$$

Using the flow property of $\nu_{(M, \mathcal{F})}$ we observe that af $(a)$ is a $\mathcal{F}_{\text {ol-natural }}$ affinor on $F$.

6. Natural operators lifting infinitesimal automorphisms of foliated manifolds to vector fields on product preserving bundles. Let $F: \mathcal{F}_{o l} \rightarrow \mathcal{F} \mathcal{M}$ be a product preserving bundle functor and $\mu: A \rightarrow B$ be its Weil algebra homomorphism. Let $p \geq 1$ and $q \geq 1$ be integers.

A $\mathcal{F}_{o l} l_{p, q^{-}}$natural operator $C$ transforming infinitesimal automorphisms $X$ of $\mathcal{F}_{o l} l_{p, q}$-objects $(M, \mathcal{F})$ into vector fields $C(X)$ on $F(M, \mathcal{F})$ is a family of regular $\mathcal{F}_{o l}, q^{-}$invariant operators

$$
C_{(M, \mathcal{F})}: \mathcal{X}(M, \mathcal{F}) \rightarrow \mathcal{X}(F(M, \mathcal{F}))
$$

from the space $\mathcal{X}(M, \mathcal{F})$ of all infinitesimal automorphisms of $(M, \mathcal{F})$ into the space $\mathcal{X}(F(M, \mathcal{F}))$ of all vector fields on $F(M, \mathcal{F})$ for any $\mathcal{F}_{o l} l_{p, q}$-objects $(M, \mathcal{F})$. The $\mathcal{F}_{o l} l_{p, q}$-invariance means that for any $\mathcal{F}_{o l} l_{p, q}$-map $\varphi:\left(M_{1}, \mathcal{F}_{1}\right) \rightarrow$ $\left(M_{2}, \mathcal{F}_{2}\right)$ and any infinitesimal automorphisms $X_{1} \in \mathcal{X}\left(M_{1}, \mathcal{F}_{1}\right)$ and $X_{2} \in$ $\mathcal{X}\left(M_{2}, \mathcal{F}_{2}\right)$, if $X_{1}$ and $X_{2}$ are $\varphi$-related then $C\left(X_{1}\right)$ and $C\left(X_{2}\right)$ are $F \varphi$ related. The regularity means that $C$ transforms smoothly parametrized families of infinitesimal automorphisms into smoothly parametrized families of vector fields.

EXAMPLE 7 (The flow operator). Let $(M, \mathcal{F})$ be a $\mathcal{F}_{o l_{p, q}}$ object. Let $X \in$ $\mathcal{X}(M, \mathcal{F})$. Then the flow $\operatorname{Exp}(t X)$ of $X$ is formed by $\mathcal{F}_{o l} l_{p, q}$-maps. So we can apply a functor $F: \mathcal{F}_{o l} \rightarrow \mathcal{F} \mathcal{M}$ to $\operatorname{Exp}(t X)$ and obtain the flow $F(\operatorname{Exp}(t X))$ on $F(M, \mathcal{F})$. Then we have the vector field $\mathcal{F} X$ on $F(M, \mathcal{F})$ corresponding to the flow $F(\operatorname{Exp}(t X))$. Clearly, the correspondence $\mathcal{F}: X \rightarrow \mathcal{F} X$ is a $\mathcal{F}_{o} l_{p, q}$-natural operator in question.

The main result of this note is the following classification theorem.

ThEOREM 3. Let $F: \mathcal{F}$ ol $\rightarrow \mathcal{F M}$ be a product preserving bundle functor and $\mu: A \rightarrow B$ be its Weil algebra homomorphism. Let $p \geq 1$ and $q \geq 1$

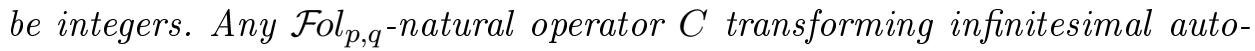
morphisms $X \in \mathcal{X}(M, \mathcal{F})$ into vector fields $C(X) \in \mathcal{X}(F(M, \mathcal{F}))$ is of the form

$$
C(X)=\operatorname{af}(a) \circ \mathcal{F} X+\text { op }(D)
$$

for some unique $a \in A$ and $D \in \operatorname{Der}(\mu)$. 
Proof. Let $C$ be an operator in question. Let $(M, \mathcal{F})$ be a $\mathcal{F}_{o l} l_{p, q}$-object. Let 0 be the zero infinitesimal automorphism on $(M, \mathcal{F})$ for any $\mathcal{F}_{o l} l_{p, q}$-object. Then $C(0)$ is a $\mathcal{F}_{o l} l_{p, q}$-canonical vector field on $F_{\mid \mathcal{F}_{o l}, q}$. Thus replacing $C$ by $C-C(0)$ and applying Proposition 6 we can assume that $C(0)=0$.

Since any automorphism $X$ on $(M, \mathcal{F})$ with non-vanishing transversal vector field is $\partial / \partial x^{1}$ on $\left(M_{0}, \mathcal{F}_{0}\right)=\left(\mathbb{R}^{q} \times \mathbb{R}^{p},\left\{\{a\} \times \mathbb{R}^{p}\right\}_{a \in \mathbb{R}^{q}}\right)$ in some adapted coordinates, $C$ is uniquely determined by $C\left(\varrho \partial / \partial x^{1}\right): A^{q} \times B^{p} \rightarrow$ $A^{q} \times B^{p}, \varrho \in \mathbb{R}$. Using the invariance with respect to the homotheties being $\mathcal{F}_{o l} l_{p}$-morphisms $\left(M_{0}, \mathcal{F}_{0}\right) \rightarrow\left(M_{0}, \mathcal{F}_{0}\right)$ and the homogeneous function theorem $([2])$ and $C(0)=0$ we deduce that for any $\varrho$ the map $C\left(\varrho \frac{\partial}{\partial x^{1}}\right)$ : $A^{q} \times B^{p} \rightarrow A^{q} \times B^{p}$ is constant and linearly dependent on $\varrho$. Then using the invariance with respect to $\mathcal{F}_{o l} l_{p, q^{-}}$maps $\left(x^{1}, t x^{2}, \ldots, t x^{q}, t y^{1}, \ldots, t y^{p}\right)$ for $t \neq 0$ we deduce that the map $C\left(\varrho \partial / \partial x^{1}\right): A^{q} \times B^{p} \rightarrow A \times\{0\} \times\{0\}$ is constant and linearly dependent on $\varrho$. Hence the vector space of all natural operators $C$ in question with $C(0)=0$ is at most $\operatorname{dim}_{\mathbb{R}} A$-dimensional. But all natural operators af $(a) \circ \mathcal{F}$ form a $\operatorname{dim}_{\mathbb{R}} A$-dimensional vector space. Thus the proof is complete by a dimension argument.

REMARK 3. Let $\mathcal{F M}_{q, p}$ be the category of fibered manifolds with $q$ dimensional bases and $p$-dimensional fibers and their local fibered diffeomorphisms. The category $\mathcal{F} \mathcal{M}_{q, p}$ is in an obvious way a subcategory in $\mathcal{F}_{o l} l_{p, q}$. The categories $\mathcal{F M}_{q, p}$ and $\mathcal{F}_{o l} l_{p, q}$ have the same skeleton. Any projectable vector field $X$ on an $\mathcal{F M}_{q, p^{-o b j e c t}} Y$ is in an obvious way an infinitesi-

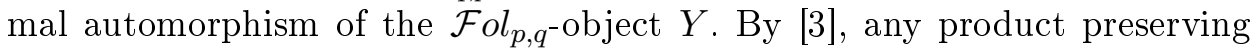
bundle functor $F: \mathcal{F M} \rightarrow \mathcal{F} \mathcal{M}$ can be uniquely extended to a product preserving bundle functor $F: \mathcal{F}_{o l} \rightarrow \mathcal{F} \mathcal{M}$. In [4], J. Tomáš classified all $\mathcal{F} \mathcal{M}_{q, p}$-natural operators $C$ transforming projectable vector fields $X$ on an $\mathcal{F} \mathcal{M}_{q, p^{-}}$object $Y$ into vector fields $C(X)$ on $F Y$ for any product preserving bundle functor $F: \mathcal{F M} \rightarrow \mathcal{F M}$ and deduced the corresponding formula $C(X)=a f(a) \circ \mathcal{F} X+o p(D)$. Therefore it seems that Theorem 3 can also be deduced from the result of [4].

7. An application to the normal bundle. Let $N: \mathcal{F}_{o l} l_{p, q} \rightarrow \mathcal{F} \mathcal{M}$ be the normal bundle functor (see Introduction). Its corresponding Weil algebra homomorphism is the unique algebra homomorphism $\kappa_{\mathbb{D}}: \mathbb{D} \rightarrow \mathbb{R}$, where $\mathbb{D}$ is the algebra of dual numbers (see the text after Example 2). It is easily seen (see [2]) that $\operatorname{dim}_{\mathbb{R}} \mathbb{D}=2$ and $\operatorname{dim}_{\mathbb{R}} \operatorname{Der}\left(\kappa_{\mathbb{D}}\right)=1$. Therefore (because of Theorem 3$)$ the vector space of all $\mathcal{F}_{o l} l_{p, q}$-natural operators transforming infinitesimal automorphisms $X \in \mathcal{X}(M, \mathcal{F})$ into vector fields $C(X) \in \mathcal{X}(N(M, \mathcal{F}))$ is of dimension 3. On the other hand, given $X \in \mathcal{X}(M, \mathcal{F})$ we have the following vector fields on the (vector) normal bundle $N(M, \mathcal{F})$ :

1. The flow vector field $\mathcal{N} X$ of $X$ on $N(M, \mathcal{F})$. 
2. The vertical lifting $X^{V}$ of $X$ to $N(M, \mathcal{F})$ defined as follows. Given $v \in N_{x}(M, \mathcal{F}), x \in M$, we have $[X(x)] \in N_{x}(M, \mathcal{F})=T_{x} M / T_{x} \mathcal{F}$. Then we put $X^{V}(v):=[X(x)] \in N_{x}(M, \mathcal{F})=T_{v}\left(N_{x}(M, \mathcal{F})\right) \subset$ $T_{v}(N(M, \mathcal{F}))$.

3. The Liouville vector field $L$ on the vector bundle $N(M, \mathcal{F}), L(v)=$ $[v+t v] \in T_{v}\left(N_{x}(M, F)\right), v \in N_{x}(M, \mathcal{F}), x \in M$.

Thus we have the corresponding natural operators: $\mathcal{N},()^{V}$ and $L$. These operators are linearly independent. So (by a dimension argument) they form a basis in the vector space of all $\mathcal{F}_{0} l_{p, q}$-natural operators $C$ transforming infinitesimal automorphisms $X \in \mathcal{X}(M, \mathcal{F})$ into vector fields $C(X)$ $\in \mathcal{X}(N(M, \mathcal{F}))$. Then we have

Corollary 3. Any $\mathcal{F}_{\text {ol }}$,q-natural operator $C$ transforming infinitesimal automorphisms $X \in \mathcal{X}(M, \mathcal{F})$ into vector fields $C(X)$ on the normal bundle $N(M, \mathcal{F})$ is of the form

$$
C(X)=a \mathcal{N} X+b X^{V}+c L
$$

for some real numbers $a, b, c$.

\section{References}

[1] I. Kolár̆, On the natural operators on vector fields, Ann. Global Anal. Geom. 6 (1988), 109-117.

[2] I. Kolář, P. W. Michor and J. Slovák, Natural Operations in Differential Geometry, Springer, 1993.

[3] W. M. Mikulski, Product preserving bundles on foliated manifolds, Ann. Polon. Math. 84 (2004), 67-74.

[4] J. Tomáš, Natural operators transforming projectable vector fields to product preserving bundles, Rend. Circ. Mat. Palermo (2) Suppl. 59 (1999), 181-187.

[5] R. A. Wolak, On transverse structures of foliations, ibid. 9 (1985), 227-243.

Institute of Mathematics

Maria Curie-Skłodowska University

Pl. M. Curie-Skłodowskiej 1

20-031 Lublin, Poland

E-mail: kurek@golem.umcs.lublin.pl
Institute of Mathematics
Jagiellonian University
Reymonta 4
30-059 Kraków, Poland
ail: mikulski@im.uj.edu.pl

Received 25.8.2006

and in final form 4.3.2007 